\title{
Editorial
}

\section{Preregistration and increased transparency will benefit science}

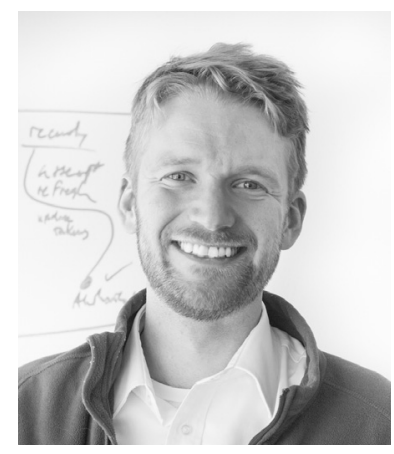

Science advances by improving existing explanations and by creating new ones. The process of refining our knowledge about how the universe works is one in which models are proposed, tested, and refined. Confirming these explanations is distinct from generating new ones. ${ }^{1}$ Conflating these two processes, confirmation and exploration, is easy to do. It is also rewarded in scientific communication because it makes an explanation appear to be more credible than it really is. This is so because when we use data to help make an explanation (a hypothesis) and then use that same data to confirm that hypothesis, we fall victim to circular reasoning that invalidates the test. Preregistration specifies in advance how data will be analyzed and thus separates the process of making an explanation from the process of confirming that explanation.

Another common problem is a lack of transparency in how science is conducted. Sharing data, analysis code, or detailed methodologies is still the exception, not the rule. While few casual readers value such details, they are essential for truly understanding, replicating, or building upon any scientific finding.

Finally, systematic evaluation of any scientific claim is difficult or impossible because of the well known bias against publishing null results. ${ }^{2}$ This bias results in a body of literature that does not accurately represent what we know. ${ }^{3}$

The combination of these three problems, conflating exploration and confirmation, incomplete methods reporting, and biased reporting of results, results in a body of scientific evidence that is less reproducible than it should be. ${ }^{4-8}$ Conducting direct replications of published studies is difficult, because there is little reward in doing so. Furthermore, replication studies that do not have similar findings to the original article create tension between the original and replication results. However, direct replications are the best way to evaluate the credibility of any scientific claim. ${ }^{9}$ Having a productive conversation about scientific claims cannot take place without more openness into the process of science and removing bias from the publication workflow.

Increasing the transparency of science requires change, which is a slow process. However, there are easy first steps that we can take, and the Transparency and Openness Promotion (TOP) Guidelines ${ }^{10}$ provide this map. The TOP Guidelines consist of eight policies that can be applied by journals, publishers, and funders. Each standard is modular and can be implemented independent of any other standard. The standards include language for data citation principles, data, materials, and code transparency, reporting guidelines, preregistration, and replication.

Crucially, each standard is tiered into three levels of increasing rigour. This simultaneously lowers barriers to adoption, while providing recommendations for future, more ideal actions. At the first level, journals can require disclosure of transparent research practices. In this level, a researcher simply must state whether or not data, for example, are available, and if so, provide a link to an appropriate data repository. Higher levels of TOP policies either mandate transparency (for example, require data sharing when ethically possible) or include independent verification (for example, verification of the dataset during peer review). The complete policy language for each standard is available and free to use at https://cos.io/top.

Preregistration is one standard that is not widely practiced outside of the clinical sciences. Fears about preregistration are that it is overly burdensome ${ }^{11}$ or devalues exploratory research. ${ }^{12}$ Transparent reporting of preregistered research with clear delineation of any unexpected, unregistered analyses address the latter fear. ${ }^{13}$ As for the former, building an easy to use workflow can help the researcher plan their study while simultaneously creating a persistent registration. Better planning puts some more work upfront, but pays dividends later on. This is what we created as part of our ongoing education campaign, the Preregistration Challenge. ${ }^{14}$ The workflow, available on the Open Science Framework (OSF) at https://osf.io/prereg, walks researchers through the creation of a completely pre-specified study and analysis plan. Preregistrations can be made public immediately, or entered into an embargo of up to four years. Publicly available preregistrations are discoverable through the OSF Registry.

There is one solution that builds upon preregistrations and removes bias from the publication process. Registered Reports ${ }^{15}$ are a publishing format in which a preregistration is submitted for peer review and improvement before conducting the study. ${ }^{16,17}$ These preregistrations, which are essentially proposals with very detailed analysis plans, are initially evaluated on 1) the importance of the questions to the field, and 2) the ability of the proposed methods and analyzes to address those questions. Only if both of those conditions are met, a study is granted in principle acceptance' (IPA) before results are known. The final publication decision relies on a second, shorter round of peer review where adherence to the accepted protocol and the successful completion of any outcome-independent quality checks (eg positive controls or manipulation checks) is evaluated. Those quality checks ensure that the study was conducted in a competent manner, in order to ensure that 
any null results are more likely to be 'true negatives'. Final studies are published without regard to perceived impact or significance, which are not relevant to the scientific validity of any finding.

Finally, a common concern about implementing new policies is the burden placed on authors and journals for compliance with new mandates. Fortunately, there is an easy way to change behaviour without the burden of regulatory oversight. Allowing researchers to signal to their peers adherence to ideal scientific practices is effective at spurring behaviour change. Open Science Badges ${ }^{18}$ are effective at increasing transparency ${ }^{19}$ and are the only evidence-based incentive programme beyond mandates that result in more data sharing. ${ }^{20}$ If complete transparency were to become part of normal scientific practice, badges may no longer be necessary. Until that utopian ideal is achieved, they are both effective and avoid the understandable hesitancy to impose a mandate.

Two goals of the open science movement are to increase clarity and decrease bias from the research workflow. Rewarding researchers for practicing behaviors that align with these values will allow science to occur as it should occur. The motto of the Royal Society, 'Nullius in verba' ('Take nobody's word for it') ${ }^{21}$ professes that evidence should be transparently evaluated. Up until recently, this was not possible. Given the ubiquity of effective tools for enabling ideal scientific practices, only our inertia is to blame for the status quo. Please take this article as a standing invitation to assist any stakeholder with implementing solutions that improve the openness, transparency, or reproducibility of science.

\section{Conflict of Interest}

DTM is an employee of the Center for Open Science (COS) (https://cos.io), a non-profit company that builds the open source Open Science Framework (OSF) (https://osf.io) and advocates for policies and practices discussed in this article.

David Mellor

Center for Open Science

david@cos.io orcid.org/0000-0002- 3125-5888

\section{References}

1 Tukey JW. We Need Both Exploratory and Confirmatory. The American Statistician. 1980 Feb;34(1):23. doi:10.2307/2682991

2 Dickersin K. The Existence of Publication Bias and Risk Factors for Its Occurrence. JAMA: The Journal of the American Medical Association. 1990 Mar 9;263(10):1385. doi:10.1001/jama.1990.03440100097014

3 Franco A, Malhotra N, Simonovits G. Publication bias in the social sciences: Unlocking the file drawer. Science. 2014 Sep 19;345(6203):1502-5. doi:10.1126/science.1255484
4 Collaboration OS. Estimating the reproducibility of psychological science. Science. 2015 Aug 28;349(6251):aac4716. doi:10.1126/science. aac4716

5 Camerer CF, Dreber A, Forsell E, et al. Evaluating replicability of laboratory experiments in economics. Science. $2016 \mathrm{Mar}$ 25;351(6280):1433-6. doi:10.1126/science.aaf0918

6 Board of Governors of the Federal Reserve System, Chang AC, Li P. Is Economics Research Replicable? Sixty Published Papers from Thirteen Journals Say “Usually Not." Finance and Economics Discussion Series. 2015 Oct;2015(83):1-26. doi:10.17016/FEDS.2015.083

7 Begley CG, Ellis LM. Drug development: Raise standards for preclinical cancer research. Nature. 2012 Mar 28;483(7391):531-3. doi:10.1038/483531a

8 Ioannidis JPA, Allison DB, Ball CA, et al. Repeatability of published microarray gene expression analyses. Nature Genetics. 2009 Feb;41(2):149-55. doi:10.1038/ng.295

9 Simons DJ. The Value of Direct Replication. Perspectives on Psychological Science. 2014 Jan;9(1):76-80. doi: $10.1177 / 1745691613514755$

10 Nosek BA, Alter G, Banks GC, et al. Promoting an open research culture. Science. 2015 Jun 26;348(6242):1422-5. doi:10.1126/science. aab2374

11 Koeing W. Striving for science that is transparent, credible-and enjoyable: a comment on Ihle et al. Behavioral Ecology. 2017 Apr;28(2). doi:https://doi.org/10.1093/beheco/arx004

12 Pre-registration would put science in chains | Times Higher Education (THE) [Internet]. Available from: https://www.timeshighereducation. com/comment/opinion/pre-registration-would-put-science-inchains/2005954.article (Accessed 29 Aug 2017)

13 Nosek B, Ebersole C, DeHaven A, et al. The Preregistration Revolution. OSF Preprint. 2017; doi:10.17605/OSF.IO/2DXU5

14 Preregistration Challenge [Internet]. Available from: https://cos.io/ prereg/ (Accessed 29Aug 2017)

15 Registered Reports [Internet]. Available from: https://cos.io/rr/ (Accessed 30 Aug 2017)

16 Chambers CD, Feredoes E, Muthukumaraswamy SD, et al. Instead of "playing the game" it is time to change the rules: Registered Reports at AIMS Neuroscience and beyond. AIMS Neuroscience. 2014 May;1(1):4-17. doi:10.3934/Neuroscience2014.1.4

17 Munafò MR, Nosek BA, Bishop DVM, et al. A manifesto for reproducible science. Nature Human Behaviour. 2017 Jan 10;1(1):21. doi:10.1038/s41562-016-0021

18 Open Science Badges [Internet]. Available from: https://cos.io/ourservices/open-science-badges/ (Accessed 29 Aug 2017)

19 Kidwell MC, Lazarević LB, Baranski E, et al. Badges to Acknowledge Open Practices: A Simple, Low-Cost, Effective Method for Increasing Transparency. PLOS Biol. 2016 May 12;14(5):e1002456. doi:10.1371/ journal.pbio. 1002456

20 Rowhani-Farid A, Allen M, Barnett AG. What incentives increase data sharing in health and medical research? A systematic review. Research Integrity and Peer Review. 2017;2:4. doi:10.1186/s41073-017-0028-9

21 History of the Royal Society [Internet]. Available from: https:// royalsociety.org/about-us/history/ (Accessed 29Aug 2017) 